\title{
Uniqueness of some differential polynomials of meromorphic functions
}

\author{
Kuldeep Singh CharaK and Banarsi LaL
}

(Received June 12, 2017)

(Revised July 1, 2018)

\begin{abstract}
In this paper, we prove some uniqueness results which improve and generalize several earlier works. Also, we prove a value distribution result concerning $f^{(k)}$ which is related to a conjecture of Fang and Wang [A note on the conjectures of Hayman, Mues and Gol'dberg, Comp. Methods, Funct. Theory (2013) 13, 533-543].
\end{abstract}

\section{Introduction}

Throughout, by a meromorphic function we always mean a non-constant meromorphic function in the complex plane $\mathbb{C}$.

We use the notations of Nevanlinna value distribution theory [2] such as $m(r, f), N(r, f), T(r, f)$ and $S(r, f)$ defined as follows:

$$
m(r, f)=m(r, \infty):=\frac{1}{2 \pi} \int_{0}^{2 \pi} \log ^{+}\left|f\left(r e^{i \theta}\right)\right| d \theta,
$$

where $r>0$ and $\log ^{+} x=\max \{\log x, 0\}$;

$$
N(r, f)=\int_{0}^{r} \frac{n(t, f)-n(0, f)}{t} d t+n(0, f) \log r
$$

where $n(t, f)$ denotes the number of poles of $f$ in $\{z:|z| \leq t\}$, each pole is counted according to its multiplicity;

$$
T(r, f)=m(r, f)+N(r, f)
$$

and $S(r, f)$ is any quantity satisfying

$$
\lim _{r \rightarrow \infty} \frac{S(r, f)}{T(r, f)}=0,
$$

possibly outside a set of finite linear measure.

2010 Mathematics Subject Classification. Primary 30D35, 30D30.

Key words and phrases. Meromorphic functions, small functions, sharing of values, Nevanlinna theory. 
By $E(a, f)$, we denote the set of zeros of $f-a$ counting multiplicities $(\mathrm{CM})$ and by $\bar{E}(a, f)$, the set of zeros of $f-a$ ignoring multiplicities (IM). Two meromorphic functions $f$ and $g$ are said to share the value $a \mathrm{CM}$ if $E(a, f)=E(a, g)$ and to share the value $a \mathrm{IM}$ if $\bar{E}(a, f)=\bar{E}(a, g)$. Further, by $E_{k)}(a, f)$, we denote the set of zeros of $f-a$ with multiplicities at most $k$ in which each zero is counted according to its multiplicity. Also, by $\bar{E}_{k)}(a, f)$, we denote the set of zeros of $f-a$ with multiplicity at most $k$, counted once.

We denote by $\mathscr{A}$, the class of meromorphic functions $f$ satisfying

$$
\bar{N}(r, f)+\bar{N}\left(r, \frac{1}{f}\right)=S(r, f) .
$$

Clearly, each member of class $\mathscr{A}$ is a transcendental meromorphic function. Also for any $a \in \mathbb{C}$, we define

$$
N_{1}\left(r, \frac{1}{f-a}\right)=N\left(r, \frac{1}{f-a}\right)-\bar{N}\left(r, \frac{1}{f-a}\right)
$$

and

$$
N_{2}\left(r, \frac{1}{f-a}\right)=\bar{N}\left(r, \frac{1}{f-a}\right)+\bar{N}_{(2}\left(r, \frac{1}{f-a}\right),
$$

where $N_{(k}(r, 1 /(f-a))$ is the counting function of those zeros of $f-a$ whose multiplicity is at least $k$, and $\bar{N}_{(k}(r, 1 /(f-a))$ is the one corresponding to ignoring multiplicity. Finally, by $S(f)$, we denote the set of small functions of $f$; that is,

$$
S(f):=\{a \mid a \text { is meromorphic and } T(r, a)=S(r, f) \text { as } r \rightarrow \infty\} .
$$

The uniqueness theory of meromorphic functions has perfected the value distribution theory of Nevanlinna and has a vast range of applications in complex analysis. For recent developments in the uniqueness theory of meromorphic functions (sharing, weighted sharing and q-difference sharing of polynomials), one may refer to $[6,8,11]$.

In the present paper, we prove some uniqueness results which improve and generalize the works of Yang and Yi [9], Wang and Gao [5], and Huang and Huang [3]. Also, a result related to a conjecture of Fang and Wang [1] concerning value distribution of $f^{(k)}-a$, where $k \in \mathbb{N}$ and $a(\not \equiv 0, \infty)$ is a small function of $f$, is obtained.

\section{Main results}

Yang and Yi [9, Theorem 3.29, p. 197] proved the following result for class $\mathscr{A}$ : 
THEOREM A. Let $f, g \in \mathscr{A}$, and a be a non-zero complex number. Furthermore, let $k$ be a positive integer.

(i) If $\bar{E}_{1)}(a, f)=\bar{E}_{1)}(a, g)$, then $f \equiv g$ or $f g \equiv a^{2}$.

(ii) If $\bar{E}_{1)}\left(a, f^{(k)}\right)=\bar{E}_{1)}\left(a, g^{(k)}\right)$, then $f \equiv g$ or $f^{(k)} g^{(k)} \equiv a^{2}$.

A function $f$ is said to share a value a partially with $g$ IM if $\bar{E}(a, f) \subseteq$ $\bar{E}(a, g)$. We use the notation $N_{1)}(r, 1 /(f-a) \mid g \neq a)$, to denote the simple zeros of $f-a$, that are not the zeros of $g-a$. Using this notation and the notion of partial sharing, we improve Theorem $\mathrm{A}$ as

THEOREM 1. Let $f, g \in \mathscr{A}$, a be a non-zero complex number and $k$ be $a$ positive integer.

(i) If $\bar{E}_{1)}(a, f) \subseteq \bar{E}_{1)}(a, g)$ and $N_{1)}(r, 1 /(g-a) \mid f \neq a)=S(r, g)$, then $f \equiv g$ or $f g \equiv a^{2}$.

(ii) If $\bar{E}_{1)}\left(a, f^{(k)}\right) \subseteq \bar{E}_{1)}\left(a, g^{(k)}\right)$ and $N_{1)}\left(r, 1 /\left(g^{(k)}-a\right) \mid f^{(k)} \neq a\right)=S(r, g)$, then $f \equiv g$ or $f^{(k)} g^{(k)} \equiv a^{2}$.

Example. Consider $f(z)=e^{z}$ and $g(z)=e^{2 z}$. Then $f, g \in \mathscr{A}, \bar{E}_{1)}(1, f) \subseteq$ $\bar{E}_{1)}(1, g)$ and $N_{1)}(r, 1 /(g-1) \mid f \neq 1) \neq S(r, g)$, and the conclusion of Theorem 1 does not hold. Thus, the condition " $N_{1)}(r, 1 /(g-a) \mid f \neq a)=S(r, g)$ " in Theorem 1, is essential.

In 2011, Huang and Huang [3, Theorem 3, p. 231] improved a result of Yang and Hua [7, Theorem 1, p. 396] as

THEOREM B. Let $f$ and $g$ be two meromorphic functions and $n \geq 19$ be an integer. If $E_{1)}\left(1, f^{n} f^{\prime}\right)=E_{1)}\left(1, g^{n} g^{\prime}\right)$, then either $f=d g$ for some $(n+1)$-th root of unity $d$ or $f(z)=c_{1} e^{c z}$ and $g(z)=c_{2} e^{-c z}$, where $c, c_{1}, c_{2}$ are constants satisfying $\left(c_{1} c_{2}\right)^{n+1} c^{2}=-1$.

In this paper, we improve Theorem B for functions of class $\mathscr{A}$ as

TheOREM 2. Let $f, g \in \mathscr{A}, n \geq 2$ be an integer and $a(\neq 0) \in \mathbb{C}$. If $\bar{E}_{1)}\left(a, f^{n} f^{\prime}\right)=\bar{E}_{1)}\left(a, g^{n} g^{\prime}\right)$, then either $f=d g$ for some $(n+1)$-th root of unity $d$ or $f(z)=c_{1} e^{c z}$ and $g(z)=c_{2} e^{-c z}$, where $c, c_{1}, c_{2}$ are constants satisfying $\left(c_{1} c_{2}\right)^{n+1} c^{2}=-a^{2}$.

Concerning sharing of small functions, Wang and Gao [5, Theorem 1.3, p. 2] proved:

THEOREM C. Let $f$ and $g$ be two transcendental meromorphic functions, $a(\not \equiv 0) \in S(f) \cap S(g)$, and let $n \geq 11$ be a positive integer. If $f^{n} f^{\prime}$ and $g^{n} g^{\prime}$ share a $C M$, then either $f^{n} f^{\prime} g^{n} g^{\prime} \equiv a^{2}$ or $f=d g$ for some $(n+1)$-th root of unity $d$. 
DEFINITION. Let $f$ and $g$ be two non-constant meromorphic functions, and $a$ is a small function related to both $f$ and $g$. We say that $f$ and $g$ share the small function $a \mathrm{CM}$ if $f-a$ and $g-a$ assume the same zeros with the same multiplicities.

Here in this paper, we partially extend Theorem $\mathrm{C}$ to a more general class of differential polynomials as

THEOREM 3. Let $f$ and $g$ be two transcendental meromorphic functions, $a(\not \equiv 0) \in S(f) \cap S(g)$, and let $n, m, k$ be positive integers satisfying $n>k m+$ $3 m+2 k+8$, and $m>k-1$. If $f^{n}\left(f^{m}\right)^{(k)}$ and $g^{n}\left(g^{m}\right)^{(k)}$ share a $C M$, then either

$$
f^{n}\left(f^{m}\right)^{(k)} g^{n}\left(g^{m}\right)^{(k)} \equiv a^{2} \quad \text { or } \quad f^{n}\left(f^{m}\right)^{(k)} \equiv g^{n}\left(g^{m}\right)^{(k)} .
$$

For $m>k-1$, we have $n>k^{2}+4 k+5$ so that by substituting $k=1$, we get $n>10$. Thus Theorem 3 reduces to Theorem $\mathrm{C}$.

Concerning the value distribution of $k$-th derivative of a meromorphic function, Fang and Wang [1, Proposition 3, p. 542] proved the following result:

THEOREM D. Let $f$ be a transcendental meromorphic function having at most finitely many simple zeros. Then $f^{(k)}$ takes on every non-zero polynomial infinitely often for $k=1,2,3, \ldots$

Definition. A meromorphic function $f$ is said to take a function $h$ infinitely often if $f-h$ has infinitely many zeros.

Further, Fang and Wang [1, Question 2, p. 543] asked the following question:

QUESTION. Let $f$ be a transcendental meromorphic function having at most finitely many simple zeros. Must $f^{(k)}$ take on every non-zero rational function infinitely often for $k=1,2,3, \ldots$ ?

Here, we obtained a result related to the above question involving small function as

THEOREM 4. Let $f$ be a transcendental meromorphic function having at most finitely many simple zeros and $N\left(r, 1 / f^{\prime \prime}\right)=S(r, f)$. Let $a(\not \equiv 0, \infty) \in$ $S(f)$, then $f^{(k)}-a$ has infinitely many zeros for $k=1,2,3, \ldots$

\section{Some lemmas}

We recall the following results which we shall use in the proof of main results of this paper: 
Lemma 1 [7, Theorem 3, p. 396]. Let $f$ and $g$ be two non-constant entire functions, $n \geq 1$ and $a(\neq 0) \in \mathbb{C}$. If $f^{n} f^{\prime} g^{n} g^{\prime}=a^{2}$, then $f(z)=c_{1} e^{c z}$ and $g(z)=c_{2} e^{-c z}$, where $c, c_{1}, c_{2}$ are constants satisfying $\left(c_{1} c_{2}\right)^{n+1} c^{2}=-a^{2}$.

Lemma $2\left[9\right.$, Lemma 1.10, p. 82]. Let $f_{1}$ and $f_{2}$ be non-constant meromorphic functions and let $c_{1}, c_{2}$ and $c_{3}$ be non-zero constants. If $c_{1} f_{1}+c_{2} f_{2} \equiv$ $c_{3}$, then

$$
T\left(r, f_{1}\right)<\bar{N}\left(r, \frac{1}{f_{1}}\right)+\bar{N}\left(r, \frac{1}{f_{2}}\right)+\bar{N}\left(r, f_{1}\right)+S\left(r, f_{1}\right) .
$$

Lemma 3 [9, Lemma 3.8, p. 193]. If $f \in \mathscr{A}$ and $k$ is a positive integer, then $f^{(k)} \in \mathscr{A}$.

Lemma 4 [9, Lemma 3.9, p. 194]. If $f, g \in \mathscr{A}$ and $f^{(k)}=g^{(k)}$, where $k$ is a positive integer, then $f \equiv g$.

Lemma 5 [9, Lemma 3.10, p. 194]. If $f \in \mathscr{A}$ and $a$ is a finite non-zero number, then

$$
N_{1)}\left(r, \frac{1}{f-a}\right)=T(r, f)+S(r, f),
$$

where $N_{1)}(r, 1 /(f-a))$ denotes the simple zeros of $f-a$.

Lemma 6 [9, Theorem 1.24, p. 39]. Suppose $f$ is a non-constant meromorphic function and $k$ is a positive integer. Then

$$
N\left(r, \frac{1}{f^{(k)}}\right) \leq N\left(r, \frac{1}{f}\right)+k \bar{N}(r, f)+S(r, f) .
$$

Lemma 7 [5, Lemma 2.3, p. 3]. Let $f$ and $g$ be two meromorphic functions. If $f$ and $g$ share $1 C M$, then one of the following must occur: i) $T(r, f)+T(r, g) \leq 2\left\{N_{2}(r, 1 / f)+N_{2}(r, 1 / g)+N_{2}(r, f)+N_{2}(r, g)\right\}+S(r, f)+$ $S(r, g)$, ii) either $f \equiv g$ or $f g \equiv 1$.

Lemma 8 [1, Lemma 1, p. 537]. Let $f$ be a transcendental meromorphic function, let $k \geq 2$ be an integer, and $\varepsilon>0$. Then

$$
(k-1) \bar{N}(r, f)+N_{1}\left(r, \frac{1}{f}\right) \leq N\left(r, \frac{1}{f^{(k)}}\right)+\varepsilon T(r, f) .
$$

\section{Proof of main results}

We divide this section into four subsections as follows: 
4.1. Proof of Theorem 1. Since $\bar{E}_{1)}(a, f) \subseteq \bar{E}_{1)}(a, g)$,

$$
N_{1)}\left(r, \frac{1}{f-a}\right) \leq N_{1)}\left(r, \frac{1}{g-a}\right) \text {. }
$$

Since (by Lemma 5)

$$
N_{1)}\left(r, \frac{1}{f-a}\right)=T(r, f)+S(r, f)
$$

and

$$
N_{1)}\left(r, \frac{1}{g-a}\right)=T(r, g)+S(r, g) \text {, }
$$

therefore,

$$
\begin{aligned}
& N_{(2}\left(r, \frac{1}{f-a}\right)=S(r, f), \\
& N_{(2}\left(r, \frac{1}{g-a}\right)=S(r, g)
\end{aligned}
$$

and

$$
T(r, g) \geq T(r, f)+S(r, f) .
$$

Define a function $h: \mathbb{C} \rightarrow \overline{\mathbb{C}}$ by

$$
h(z)=\frac{f(z)-a}{g(z)-a} .
$$

Since $\bar{E}_{1)}(a, f) \subseteq \bar{E}_{1)}(a, g)$, we have

$$
\begin{gathered}
\bar{N}(r, h) \leq \bar{N}(r, f)+\bar{N}_{(2}\left(r, \frac{1}{g-a}\right)+N_{1)}\left(r, \frac{1}{g-a} \mid f \neq a\right)=S(r, g) \\
\bar{N}\left(r, \frac{1}{h}\right) \leq \bar{N}(r, g)+\bar{N}_{(2}\left(r, \frac{1}{f-a}\right)=S(r, g)
\end{gathered}
$$

and

$$
T(r, h) \leq T(r, f)+T(r, g)+O(1) \leq 2 T(r, g)+S(r, g) .
$$

Let $f_{1}=(1 / a) f, f_{2}=h, f_{3}=(-1 / a) h g$. Then,

$$
\sum_{j=1}^{3} f_{j} \equiv 1 .
$$


Combining (2), (3) and (4), we get

$$
\sum_{j=1}^{3}\left(\bar{N}\left(r, f_{j}\right)+\bar{N}\left(r, \frac{1}{f_{j}}\right)\right)=S(r, g) .
$$

Clearly, $f_{1}, f_{2}$ and $f_{3}$ are linearly dependent and so there exist three constants $c_{1}, c_{2}$ and $c_{3}$ (at least one of them is not zero) such that

$$
\sum_{j=1}^{3} c_{j} f_{j}=0
$$

If $c_{1}=0$, then from (6) we see that $c_{2} \neq 0, c_{3} \neq 0$, and

$$
f_{3}=-\frac{c_{2}}{c_{3}} f_{2}
$$

Substituting (7) into (5) gives

$$
f_{1}+\left(1-\frac{c_{2}}{c_{3}}\right) f_{2}=1
$$

From (7) and (8), we get

$$
T\left(r, f_{3}\right)=T\left(r, f_{1}\right)+O(1)
$$

and thus

$$
T(r)=T\left(r, f_{1}\right)+O(1),
$$

where $T(r)=\max _{1 \leq j \leq 3}\left\{T\left(r, f_{j}\right)\right\}$.

Since $f_{1}$ is not a constant, it follows from (8) that $1-c_{2} / c_{3} \neq 0$. From (8), (9) and Lemma 2, we deduce that

$$
T(r)<\bar{N}\left(r, \frac{1}{f_{1}}\right)+\bar{N}\left(r, \frac{1}{f_{2}}\right)+\bar{N}\left(r, f_{1}\right)+S(r)=S(r),
$$

where $S(r)=o(T(r))$, which is a contradiction and so $c_{1} \neq 0$, and then (6) gives

$$
f_{1}=-\frac{c_{2}}{c_{1}} f_{2}-\frac{c_{3}}{c_{1}} f_{3}
$$

Now, from (5) and (10), we get

$$
\left(1-\frac{c_{2}}{c_{1}}\right) f_{2}+\left(1-\frac{c_{3}}{c_{1}}\right) f_{3}=1 .
$$

We consider the following three cases: 
Case 1: $1-c_{2} / c_{1} \neq 0$ and $1-c_{3} / c_{1} \neq 0$. In this case, (10) and (11) give

$$
f_{1}=\frac{c_{2}-c_{3}}{c_{1}-c_{2}} f_{3}-\frac{c_{2}}{c_{1}-c_{2}} .
$$

From (11) and (12), we have

$$
T\left(r, f_{2}\right)=T\left(r, f_{1}\right)+O(1)
$$

and hence

$$
T(r)=T\left(r, f_{1}\right)+O(1) .
$$

Applying Lemma 2 to (11) and using (13), we obtain

$$
T(r)<\bar{N}\left(r, \frac{1}{f_{2}}\right)+\bar{N}\left(r, \frac{1}{f_{3}}\right)+\bar{N}\left(r, f_{2}\right)+S(r)=S(r),
$$

which is a contradiction.

Case 2: $1-c_{2} / c_{1}=0$. From (11), we have $1-c_{3} / c_{1} \neq 0$, and

$$
f_{3}=\frac{c_{1}}{c_{1}-c_{3}}
$$

Since $1-c_{2} / c_{1}=0$, we obtain $c_{1}=c_{2}$. Thus from (10) and (14), we obtain

$$
f_{1}+f_{2}=-\frac{c_{3}}{c_{1}-c_{3}}
$$

If $c_{3} \neq 0$, then by applying Lemma 2 to $(15)$, we obtain

$$
T(r)<\bar{N}\left(r, \frac{1}{f_{1}}\right)+\bar{N}\left(r, \frac{1}{f_{2}}\right)+\bar{N}\left(r, f_{1}\right)+S(r)=S(r),
$$

which is a contradiction. Hence $c_{3}=0$ and so from (14), it follows that $f_{3} \equiv 1$.

Case 3: $1-c_{3} / c_{1}=0$. From (11), we have $1-c_{2} / c_{1} \neq 0$, and

$$
f_{2}=\frac{c_{1}}{c_{1}-c_{2}}
$$

Since $1-c_{3} / c_{1}=0$, we obtain $c_{1}=c_{3}$. Thus from (10) and (16), we obtain

$$
f_{1}+f_{3}=-\frac{c_{2}}{c_{1}-c_{2}} .
$$


If $c_{2} \neq 0$, then by applying Lemma 2 to $(17)$, we obtain

$$
T(r)<\bar{N}\left(r, \frac{1}{f_{1}}\right)+\bar{N}\left(r, \frac{1}{f_{3}}\right)+\bar{N}\left(r, f_{1}\right)+S(r)=S(r),
$$

which is a contradiction. Hence $c_{2}=0$ and so from (16), it follows that $f_{2} \equiv 1$.

Thus if $f_{2} \equiv 1$, then by (2), we get $f \equiv g$. If $f_{3} \equiv 1$, then (2) gives $f g \equiv a^{2}$. This proves (i).

From Lemma 3, we see that $f^{(k)}, g^{(k)} \in \mathscr{A}$. Using the conclusion of (i), we get either

$$
f^{(k)} \equiv g^{(k)}
$$

or

$$
f^{(k)} g^{(k)} \equiv a^{2}
$$

If $f^{(k)} \equiv g^{(k)}$, then from Lemma 4, we have $f \equiv g$. This completes the proof of (ii).

4.2. Proof of Theorem 2. Let the functions $F$ and $G$ be given by

$$
F=\frac{f^{n+1}}{n+1} \quad \text { and } \quad G=\frac{g^{n+1}}{n+1} .
$$

By hypothesis, $\bar{E}_{1)}\left(a, f^{n} f^{\prime}\right)=\bar{E}_{1)}\left(a, g^{n} g^{\prime}\right)$, therefore

$$
\bar{E}_{1)}\left(a, F^{\prime}\right)=\bar{E}_{1)}\left(a, G^{\prime}\right) \text {. }
$$

Now

$$
\begin{aligned}
\bar{N}(r, F)+\bar{N}\left(r, \frac{1}{F}\right) & =\bar{N}\left(r, \frac{f^{n+1}}{n+1}\right)+\bar{N}\left(r, \frac{n+1}{f^{n+1}}\right) \\
& =\bar{N}(r, f)+\bar{N}\left(r, \frac{1}{f}\right) \\
& =S(r, f) \\
& =S(r, F) .
\end{aligned}
$$

Similarly by replacing $F$ by $G$ in above equation, we have

$$
\bar{N}(r, G)+\bar{N}\left(r, \frac{1}{G}\right)=S(r, G) .
$$

Thus $F, G \in \mathscr{A}$ and so by the Theorem 2.1, it follows that either

$$
F^{\prime} G^{\prime} \equiv a^{2} \quad \text { or } \quad F \equiv G .
$$


Consider the case $F^{\prime} G^{\prime} \equiv a^{2}$, that is,

$$
f^{n} f^{\prime} g^{n} g^{\prime} \equiv a^{2}
$$

Suppose that $z_{1}$ is a pole of $f$ of order $p$. Then $z_{1}$ is a zero of $g$ of order say $q$ and so from (18), we find that

$$
n q+q-1=n p+p+1 .
$$

That is, $(q-p)(n+1)=2$, which is not possible as $n \geq 2$ and $p, q$ are positive integers. Thus $f$ and $g$ are entire functions and so from Lemma 1, we get $f(z)=c_{1} e^{c z}$ and $g(z)=c_{2} e^{-c z}$, where $c, c_{1}, c_{2}$ are constants satisfying $\left(c_{1} c_{2}\right)^{n+1} c^{2}=-a^{2}$.

Next consider the case when $F \equiv G$. This gives

$$
\frac{f^{n+1}}{n+1}=\frac{g^{n+1}}{n+1}
$$

or

$$
f^{n+1}=g^{n+1} .
$$

Hence $f=d g$ for some $(n+1)$-th root of unity $d$.

4.3. Proof of Theorem 3. Let the functions $F$ and $G$ be given by

$$
F=\frac{f^{n}\left(f^{m}\right)^{(k)}}{a} \quad \text { and } \quad G=\frac{g^{n}\left(g^{m}\right)^{(k)}}{a} .
$$

Since $f^{n}\left(f^{m}\right)^{(k)}$ and $g^{n}\left(g^{m}\right)^{(k)}$ share $a$ CM, $F$ and $G$ share 1 CM. Since (by Lemma 6 and $T(r, a)=S(r, f))$,

$$
\begin{aligned}
N_{2}\left(r, \frac{1}{F}\right)+N_{2}(r, F) & \leq N_{2}\left(r, \frac{1}{f^{n}\left(f^{m}\right)^{(k)}}\right)+N_{2}\left(r, f^{n}\left(f^{m}\right)^{(k)}\right)+S(r, f) \\
& \leq N_{2}\left(r, \frac{1}{f^{n}}\right)+N_{2}\left(r, \frac{1}{\left(f^{m}\right)^{(k)}}\right)+2 \bar{N}\left(r, f^{n}\left(f^{m}\right)^{(k)}\right)+S(r, f) \\
& \leq 2 \bar{N}\left(r, \frac{1}{f}\right)+N\left(r, \frac{1}{\left(f^{m}\right)^{(k)}}\right)+2 \bar{N}(r, f)+S(r, f) \\
& \leq 2 \bar{N}\left(r, \frac{1}{f}\right)+N\left(r, \frac{1}{f^{m}}\right)+k \bar{N}\left(r, f^{m}\right)+2 \bar{N}(r, f)+S(r, f) \\
& =2 \bar{N}\left(r, \frac{1}{f}\right)+m N\left(r, \frac{1}{f}\right)+k \bar{N}(r, f)+2 \bar{N}(r, f)+S(r, f)
\end{aligned}
$$




$$
\begin{aligned}
& =2 \bar{N}\left(r, \frac{1}{f}\right)+m N\left(r, \frac{1}{f}\right)+(k+2) \bar{N}(r, f)+S(r, f) \\
& \leq 2 T(r, f)+m T(r, f)+(k+2) T(r, f)+S(r, f) \\
& =(k+m+4) T(r, f)+S(r, f),
\end{aligned}
$$

therefore,

$$
N_{2}\left(r, \frac{1}{F}\right)+N_{2}(r, F) \leq(k+m+4) T(r, f)+S(r, f) .
$$

On the similar lines we can write (19) for the function $G$ as

$$
N_{2}\left(r, \frac{1}{G}\right)+N_{2}(r, G) \leq(k+m+4) T(r, g)+S(r, g) .
$$

Since

$$
\begin{aligned}
n T(r, f)=T\left(r, f^{n}\right) & =T\left(r, \frac{f^{n}\left(f^{m}\right)^{(k)}}{a} \cdot \frac{a}{\left(f^{m}\right)^{(k)}}\right) \\
& \leq T(r, F)+T\left(r, \frac{1}{\left(f^{m}\right)^{(k)}}\right)+T(r, a)+S(r, f) \\
& \leq T(r, F)+T\left(r, \frac{1}{\left(f^{m}\right)^{(k)}}\right)+S(r, f) \\
& \leq T(r, F)+(k+1) T\left(r, \frac{1}{f^{m}}\right)+S(r, f) \\
& =T(r, F)+(k m+m) T\left(r, \frac{1}{f}\right)+S(r, f),
\end{aligned}
$$

therefore

$$
(n-k m-m) T(r, f) \leq T(r, F)+S(r, f) .
$$

Similarly,

$$
(n-k m-m) T(r, g) \leq T(r, G)+S(r, g) .
$$

Adding (21) and (22), we get

$$
(n-k m-m)\{T(r, f)+T(r, g)\} \leq\{T(r, F)+T(r, G)\}+S(r, f)+S(r, g) \text {. }
$$

Suppose that 


$$
\begin{aligned}
T(r, F)+T(r, G) \leq & 2\left\{N_{2}\left(r, \frac{1}{F}\right)+N_{2}\left(r, \frac{1}{G}\right)+N_{2}(r, F)+N_{2}(r, G)\right\} \\
& +S(r, F)+S(r, G)
\end{aligned}
$$

holds. Then from (19), (20), (23) and (24), we have

$$
\begin{aligned}
(n- & k m-m)\{T(r, f)+T(r, g)\} \\
\leq & 2\left\{N_{2}\left(r, \frac{1}{F}\right)+N_{2}\left(r, \frac{1}{G}\right)+N_{2}(r, F)+N_{2}(r, G)\right\} \\
& +S(r, f)+S(r, g) \\
\leq & 2(k+m+4)\{T(r, f)+T(r, g)\}+S(r, f)+S(r, g) \\
= & (2 k+2 m+8)\{T(r, f)+T(r, g)\}+S(r, f)+S(r, g),
\end{aligned}
$$

which implies that

$$
(n-k m-3 m-2 k-8)\{T(r, f)+T(r, g)\} \leq S(r, f)+S(r, g),
$$

a contradiction since $n>k m+3 m+2 k+8$, where $m>k-1$.

Thus, by Lemma 7, it follows that either

$$
F G \equiv 1
$$

or

$$
F \equiv G
$$

That is, either

$$
f^{n}\left(f^{m}\right)^{(k)} g^{n}\left(g^{m}\right)^{(k)} \equiv a^{2}
$$

or

$$
f^{n}\left(f^{m}\right)^{(k)}=g^{n}\left(g^{m}\right)^{(k)} .
$$

\subsection{Proof of Theorem 2. Since}

$$
\begin{aligned}
m\left(r, \frac{1}{f}\right) & =m\left(r, \frac{f^{(k)}}{f} \cdot \frac{1}{f^{(k)}}\right) \\
& \leq m\left(r, \frac{1}{f^{(k)}}\right)+m\left(r, \frac{f^{(k)}}{f}\right) \\
& =m\left(r, \frac{1}{f^{(k)}}\right)+S(r, f),
\end{aligned}
$$


therefore,

$$
T(r, f)-N\left(r, \frac{1}{f}\right) \leq T\left(r, f^{(k)}\right)-N\left(r, \frac{1}{f^{(k)}}\right)+S(r, f),
$$

and so

$$
N\left(r, \frac{1}{f^{(k)}}\right) \leq T\left(r, f^{(k)}\right)-T(r, f)+N\left(r, \frac{1}{f}\right)+S(r, f) .
$$

Applying the second fundamental theorem of Nevanlinna [2, Theorem 2.5, p. 47] to the function $f^{(k)}$, we get

$$
T\left(r, f^{(k)}\right) \leq \bar{N}\left(r, f^{(k)}\right)+\bar{N}\left(r, \frac{1}{f^{(k)}}\right)+\bar{N}\left(r, \frac{1}{f^{(k)}-a}\right)+S\left(r, f^{(k)}\right) .
$$

That is,

$$
T\left(r, f^{(k)}\right) \leq \bar{N}(r, f)+\bar{N}\left(r, \frac{1}{f^{(k)}}\right)+\bar{N}\left(r, \frac{1}{f^{(k)}-a}\right)+S(r, f) .
$$

Since $N\left(r, 1 / f^{\prime \prime}\right)=S(r, f), \quad$ it follows from Lemma 8 with $k=2$ that

$$
\begin{aligned}
\bar{N}(r, f)+N_{1}\left(r, \frac{1}{f}\right) & \leq N\left(r, \frac{1}{f^{\prime \prime}}\right)+\varepsilon T(r, f) \\
& =\varepsilon T(r, f)+S(r, f) .
\end{aligned}
$$

Thus, from (25), (26) and the fact that $f$ has finitely many simple zeros, we get

$$
\begin{aligned}
T(r, f) & \leq \bar{N}\left(r, \frac{1}{f^{(k)}-a}\right)+\bar{N}(r, f)+N\left(r, \frac{1}{f}\right)+S(r, f) \\
& \leq N\left(r, \frac{1}{f^{(k)}-a}\right)+\bar{N}(r, f)+N\left(r, \frac{1}{f}\right)+S(r, f) \\
& =N\left(r, \frac{1}{f^{(k)}-a}\right)+\bar{N}(r, f)+N_{1}\left(r, \frac{1}{f}\right)+\bar{N}\left(r, \frac{1}{f}\right)+S(r, f) \\
& \leq N\left(r, \frac{1}{f^{(k)}-a}\right)+\varepsilon T(r, f)+\frac{1}{2} N\left(r, \frac{1}{f}\right)+S(r, f)
\end{aligned}
$$




$$
\begin{aligned}
& \leq N\left(r, \frac{1}{f^{(k)}-a}\right)+\varepsilon T(r, f)+\frac{1}{2} T(r, f)+S(r, f) \\
& =N\left(r, \frac{1}{f^{(k)}-a}\right)+\left(\frac{1}{2}+\varepsilon\right) T(r, f)+S(r, f),
\end{aligned}
$$

which implies that

$$
\left(\frac{1}{2}-\varepsilon\right) T(r, f) \leq N\left(r, \frac{1}{f^{(k)}-a}\right)+S(r, f) .
$$

Taking $\varepsilon=1 / 4$ in $(27)$, we get

$$
T(r, f) \leq 4 N\left(r, \frac{1}{f^{(k)}-a}\right)+S(r, f) .
$$

Hence $f^{(k)}-a$ has infinitely many zeros for $k=1,2,3, \ldots$.

\section{Acknowledgement}

Authors express their gratitude to the anonymous refree for his/her valuable suggestions for the improvement of the paper.

\section{References}

[1] M. Fang and Y. Wang, A note on the conjectures of Hayman, Mues and Gol'dberg, Comput. Methods Funct. Theory 13 (2013), no. 4, 533-543.

[2] W. K. Hayman, Meromorphic functions, Oxford Mathematical Monographs, Clarendon Press, Oxford, 1964.

[3] H. Huang and B. Huang, Uniqueness of meromorphic functions concerning differential monomials, Appl. Math. (Irvine) 2 (2011), no. 2, 230-235.

[4] E. Mues and M. Reinders, Meromorphic functions sharing one value and unique range sets, Kodai Math. J. 18 (1995), no. 3, 515-522.

[5] S. Wang and Z. Gao, Meromorphic functions sharing a small function, Abstr. Appl. Anal. 2007, Art. ID 60718, 6 pp.

[6] K. Yamanoi, Zeros of higher derivatives of meromorphic functions in the complex plane, Proc. Lond. Math. Soc. 106 (2013), no. 3, 703-780

[7] C. C. Yang and X. Hua, Uniqueness and value-sharing of meromorphic functions, Ann. Acad. Sci. Fenn. Math. 22 (1997), no. 2, 395-406.

[8] P. Yang and X. Liu, Value distribution of the $k$-th derivatives of meromorphic functions, Adv. Pure Math. 4 (2014), 11-16.

[9] C. C. Yang and H. X. Yi, Uniqueness theory of meromorphic functions, Math. Appl. 557, Kluwer Acad. Publ., Dordrecht, 2003.

[10] H. X. Yi, Uniqueness of meromorphic functions and a question of C. C. Yang, Complex Variables Theory Appl. 14 (1990), no. 1-4, 169-176. 
[11] X. B. Zhang and H. X. Yi, On some problems of difference functions and difference equations, Bull. Malays. Math. Sci. Soc. (2) 36 (2013), no. 4, 1127-1137.

Kuldeep Singh Charak

Depertment of Mathematics

University of Jammu

Jammu-180 006

India

E-mail: kscharak7@rediffmail.com

Banarsi Lal

Depertment of Mathematics

University of Jammu

Jammu-180 006

India

E-mail: banarsiverma644@gmail.com 